\title{
Evaluation of the in vitro activity of ceftaroline and its potential against a collection of hospital-acquired methicillin-resistant Staphylococcus aureus and hospital-acquired methicillin-susceptible Staphylococcus aureus isolates recovered from various clinical samples
}

\author{
Elisabetta Nucleo, Maria Sofia Caltagirone, Aurora Piazza, Roberta Migliavacca, \\ Melissa Spalla, Riccardo Baratto, Francesca Zara, Laura Pagani \\ Dipartimento di Scienze Clinico-Chirurgiche, Diagnostiche e Pediatriche, Unità di Microbiologia e Microbiologia Clinica, \\ Università di Pavia, Pavia
}

Key words: Ceftaroline, Hospital-Acquired Methicillin-Resistant Staphylococcus aureus (HA-MRSA), HospitalAcquired Methicillin-Susceptible Staphylococcus aureus (HA-MSSA)

Valutazione dell'attività in vitro del ceftaroline e del suo potenziale verso una collezione di stipiti di Staphylococcus aureus meticillino-resistenti e meticillino-sensibili acquisiti in ambito ospedaliero ottenuti da diversi campioni clinici

\section{SUMMARY}

Aim of the study was the evaluation of the in vitro activity of ceftaroline and its potential against a collection of Hospital-Acquired Methicillin-Resistant Staphylococcus aureus (HA-MRSA) and Hospital-Acquired MethicillinSusceptible S. aureus (HA-MSSA) isolates. S. aureus isolates from outpatients were also examined. A total of 30 MSSA and 50 MRSA strains were studied. The isolates were collected during a four-year period (2006-2010) from the Pavia area (North-West of Italy). MICs and MBCs were performed in duplicate by broth dilution method (EUCAST 20I2). Susceptibility category assessments for ceftaroline was made using EUCAST 2013 breakpoints. MICs of the antibiotics included in MicroScan Pos Breakpoint Combo Type 32 Panels (Siemens) were also evaluated. Genotyping was performed by PFGE. Ceftaroline MIC values for MSSA strains ranged from $0.06 \mathrm{mg} / \mathrm{L}$ to $0.5 \mathrm{mg} / \mathrm{L}$ and from $0.125 \mathrm{mg} / \mathrm{L}$ to $2 \mathrm{mg} / \mathrm{L}$ for MRSA strains, of which $4 / 50$ exhibited a MIC value of $2 \mathrm{mg} / \mathrm{L}$. The MIC value of these 4 isolates was confirmed by macromethod. Two out of four, clonally related, belonged to hVISA category. Furthermore, MIC50 and MIC $_{90}$ were evaluated: MSSA MIC $\mathrm{C}_{50}$ value was $0.125 \mathrm{mg} / \mathrm{L}$, while $\mathrm{MIC}_{90}$ was $0.25 \mathrm{mg} / \mathrm{L} ; \mathrm{MIC}_{50}$ and $\mathrm{MIC} \mathrm{C}_{90}$ values of the MRSA were $0.5 \mathrm{mg} / \mathrm{L}$ and I mg/L, respectively. Finally, the bactericidal activity of this molecule was investigated by MBC method. Ceftaroline MIC and MBC values coincided in $96.25 \%$ of cases, only in 3 cases the MBC value was one dilution higher than MIC value. Ceftaroline demonstrated bactericidal activity against a large number of $S$. aureus, included MRSA and hVISA.

\section{INTRODUZIONE}

Ceftaroline fosamil (Teflaro, Forest Laboratories, Inc.) è un'ossiminocefalosporina di nuova generazione, ad ampio spettro e ad azione battericida, attiva verso patogeni comunitari ed ospedalieri, quali Staphylococcus aureus meticillino-resistenti (MRSA), Streptococcus pneumoniae multiresistenti e batteri Gram-negativi non-ES $\beta$ L produttori. Il suo utilizzo negli adulti (età $>18$ anni) è stato approvato, a partire da Ottobre 2010, dalla Food and Drug Administration (FDA) per il trattamento della polmonite batterica acquisita in comunità e delle infezioni cutanee acute e dei tessuti molli. Negli Stati Uniti questo antibiotico è in commercio da Marzo 2011, mentre in Europa ha ricevuto l'approvazione dall'EMA (European Medicines Agency) per le infezioni complicate di cute e tessuti molli e polmoniti acquisite in comunità nell'Agosto 2012. I criteri interpretativi di sensibilità sono forniti, a partire dal 2013, da EUCAST (Tabella 1).

Ceftaroline è stato sviluppato a partire dalla modifica strutturale della cefalosporina di quarta generazione cefozopran. La promolecola, ceftaroline

\section{Corresponding author: Elisabetta Nucleo}

Università di Pavia, Dipartimento di Scienze Clinico-Chirurgiche, Diagnostiche e Pediatriche,

Unità di Microbiologia e Microbiologia Clinica

Via Brambilla 74, 27I00 Pavia - Tel.: 0382-984I45 - Fax 0382-5484255

E-mail: elisabetta.nucleo@unipv.it 
fosamil, che contiene un gruppo fosforico al fine di aumentare la solubilità in acqua, viene rapidamente convertito nel plasma nell'agente bioattivo, ceftaroline. L'aggiunta di un anello 1.3-tiazolidinico in posizione 3' del nucleo cefalosporinico attraverso un ponte solfidrico è responsabile dell'attività anti-MRSA del ceftaroline.

Come altri $\beta$-lattamici, questa molecola reagisce chimicamente con le PBP a formare stabilmente un complesso antibiotico-enzima stabile ed inattivo e quindi blocca la biosintesi della parete cellulare. Il ceftaroline ha un'alta affinità per la PBP2a di MRSA, per le PBPs 1-3 di MSSA e $\mathrm{PBP} 2 \mathrm{x} / 2 \mathrm{a} / 2 \mathrm{~b} / 1 \mathrm{a}$ espresse da $S$. pneumoniae. Ceftaroline è efficace, inoltre, contro stipiti di $S$. aureus vancomicina-intermedi (VISA) e vancomicina-resistenti (VRSA).

L'azione è limitata verso Enterococcus faecalis, Enterococcus faecium e Corynebacterium jeikeium. Possiede attività contro $\mathrm{i}$ comuni patogeni responsabili di polmoniti acquisite in comunità come Moraxella catarrhalis ed Haemophilus influenzae, includendo anche ceppi produttori di $\beta$-lattamasi.

Inoltre è efficace verso molti Enterobatteri quali Enterobacter cloacae, Escherichia coli e Klebsiella pneumoniae. Ha bassa attività contro patogeni produttori di $\beta$-lattamasi a spettro esteso (ESBL) come TEM-3, SHV-2 e CTX-M, metallo$\beta$-lattamasi o patogeni che sovra-esprimono la beta-lattamasi AmpC cromosomica, oltre ad organismi non fermentanti come Pseudomonas spp, Stenotrophomonas maltophilia ed Acinetobacter spp. L'inefficacia verso ceppi produttori di ES $\beta \mathrm{L}$ viene superata con l'associazione ad un inibitore delle $\beta$-lattamasi (es.: acido clavulanico).

Ha un'azione limitata anche contro batteri anaerobi, mentre, similmente ad altre cefalosporine, è attivo contro batteri Gram-positivi anaerobi come Propionibacterium spp e Peptostreptococcus spp comuni colonizzatori della faringe. È anche inattivo contro bacilli anaerobi Gram-negativi come Bacteroides fragilis e Prevotella spp $(2,3)$.

Lo scopo del presente studio è stato quello di valutare l'attività in vitro del ceftaroline fosamil ed il suo potenziale contro una collezione di isolati di S. aureus MR e MS di provenienza ospedaliera e comunitaria.

\section{MATERIALI E METODI}

Nel periodo Dicembre 2011-Luglio 2012 sono stati analizzati 80 stipiti di $S$. aureus: 50/80 MRSA e 30/80 MSSA. I ceppi in esame sono stati raccolti dall'anno 2006 all'anno 2010 e provenivano da ospedali diversi. In particolare, $24 / 80$ stipiti sono stati isolati da reparti di Clinica Medica, 17/80 da ambulatori ASL, 9/80 da ambulatori ospedalieri, 8/80 da reparti Chirurgici, 8/80 da reparti di Terapia Intensiva e 14/80 da altri reparti (Figura I). Gli isolati provenivano principalmente da tamponi ferita (36/80), emocolture (18/80) e broncoaspirati (13/80) (Figura II).

Tutti i ceppi sono stati nuovamente confermati appartenere alla specie $S$. aureus mediante il sistema semi-automatizzato Microscan (Siemens, Sacramento), utilizzando i pannelli Positive Breakpoint Combo 32.

Gli stipiti sono stati seminati, presso il Laboratorio dell'Unità di Microbiologia e Microbiologia Clinica dell'Università di Pavia, sul terreno selettivo e differenziale Mannitol Salt Agar, controllati per vitalità e purezza, e nuovamente identificati dopo passaggio su terreno agar sangue mediante il sistema semi-automatizzato MicroScan (Siemens, Sacramento), utilizzando i pannelli Positive Breakpoint Combo 32.

Mediante tali pannelli è stato possibile valutare $\mathrm{i}$ valori di MIC degli antibiotici inclusi; l'interpretazione è avvenuta secondo i criteri proposti da EUCAST 2012 (5). Per tutti gli isolati in esame la Minima Concentrazione Inibente (MIC) del ceftaroline è stata saggiata mediante il test di microdiluizione in brodo, seguita dalla valutazione della Minima Concentrazione Battericida (MBC), definita come la concentrazione di antimicrobico necessaria a ridurre il numero dei microrganismi vitali del $99.9 \%$ (1).

I criteri interpretativi di riferimento utilizzati per attribuire il profilo di sensibilità di ceftaroline sono quelli proposti da EUCAST 2013: risultano sensibili gli isolati di $S$. aureus (inclusi gli MRSA) con valori di MIC $\leq 1 \mathrm{mg} / \mathrm{L}$ (6). S. aureus ATCC 25923 è sempre stato incluso come ceppo di riferimento per il controllo di qualità dei test di sensibilità in vitro. Per i ceppi che mostravano resistenza al ceftaroline (MIC > $1 \mathrm{mg} / \mathrm{L})$, la MIC è stata confermata anche con diluizione in brodo in macrometodo (1).

Per valutare la MBC si è proceduto con la semina su terreno Mannitol salt agar di $10 \mu \mathrm{l}$ di quattro diluizioni seriali di antibiotico: una corrispondente al valore di MIC e le tre diluizioni superiori, al fine di determinare la presenza di cellule vitali. Dopo incubazione in termostato a $37^{\circ} \mathrm{C}$ overnight, l'assenza o la crescita batterica stabilisce la qualità battericida del farmaco. Gli esperimenti per valutare i valori di MIC e MBC sono stati condotti in duplicato come consigliato da EUCAST (5). Sono state valutate per ceftaroline anche $i$ valori di $\mathrm{MIC}_{50}$ e $\mathrm{MIC}_{90}$ : concentrazioni di antibiotico necessarie ad inibire rispettivamente il $50 \%$ e il $90 \%$ dei microrganismi.

Gli isolati di S. aureus in studio sono stati genotipizzati mediante Pulsed-Field-Gel-Electrophoresis 
(PFGE) utilizzando il kit di reagenti Bio-Rad $\mathrm{n}^{\circ} 1$, con enzima di restrizione SmaI (Bio-Rad Laboratories, Richmond, Calif.).

Tale metodica, tramite la risoluzione dei frammenti di restrizione ottenuti dal DNA batterico, consente di comparare diversi pattern per determinare se i ceppi sono geneticamente identici o comunque di origine clonale. Le relazioni clonali sono state interpretate in accordo con i criteri proposti da Tenover et al. (4).

\section{RISULTATI}

I valori di MIC di alcuni antibiotici attivi contro $i$ batteri Gram positivi (inclusi nel pannello Pos Breakpoint Combo Panel Type 32) e del ceftaroline sono riassunti in Tabella 2 e 3.

Per i ceppi MSSA i valori di MIC del ceftaroline risultano compresi tra $0.06 \mathrm{mg} / \mathrm{L}$ e $0.5 \mathrm{mg} / \mathrm{L}$ e tra $0.125 \mathrm{mg} / \mathrm{L}$ e $2 \mathrm{mg} / \mathrm{L}$ per i 50 ceppi MRSA; di questi, 4/50 hanno mostrato un valore di MIC pari a $2 \mathrm{mg} / \mathrm{L}$. Valore confermato anche con macrometodo. I quattro stipiti meticillino-resistenti con MIC di ceftaroline pari a $2 \mathrm{mg} / \mathrm{L}$, sono stati isolati da pazienti ricoverati presso i reparti di medicina (2), ematologia e da un ambulatorio dell'ASL. Gli isolati provenivano da bronco aspirati (2), emocoltura e tampone ferita. Mostravano inoltre un profilo di multi resistenza. 2/4 stipiti, clonalmente correlati, risultavano appartenere alla categoria hVISA, ovvero mostravano una eterogenea resistenza alla vancomicina.

Sono state valutate anche le MIC in grado di inibire il $50 \%$ e il $90 \%$ dei ceppi studiati $\left(\mathrm{MIC}_{50} \mathrm{e}\right.$ $\mathrm{MIC}_{90}$ ); per i ceppi MSSA il valore di $\mathrm{MIC}_{50}$ è risultato essere pari a $0.125 \mathrm{mg} / \mathrm{L}$, mentre quello di $\mathrm{MIC}_{90}$ è risultato essere di $0.25 \mathrm{mg} / \mathrm{L}$; per gli isolati MRSA la $\mathrm{MIC}_{50} \mathrm{e}^{\mathrm{MIC}} \mathrm{C}_{90}$ evidenziata è stata di $0.5 \mathrm{mg} / \mathrm{L}$ e $1 \mathrm{mg} / \mathrm{L}$, rispettivamente. Infine è stata determinata l'attività battericida utilizzando il metodo della MBC. L'antibiotico ha dimostrato attività battericida nei confronti di un ampio numero di $S$. aureus, compresi gli isolati resistenti (MRSA, hVISA). Nel 96.25\% (77/80) dei casi, i valori di MIC e di MBC coincidevano, mentre in 3 casi era superiore di una diluizione.

\section{DISCUSSIONE}

S. aureus rappresenta uno dei patogeni più frequentemente responsabile di infezioni ospedaliere e comunitarie in tutto il mondo.

L'emergere di resistenza ad alti livelli ai beta-lattamici e recentemente la ridotta sensibilità ai glicopeptidi creano difficoltà terapeutiche.

Ceppi MRSA, la cui frequenza in Italia secondo l'ECDC è doppia rispetto alla media Europea, sono attualmente isolati non solo in ambito ospedaliero, ma anche in pazienti ambulatoriali ed in comunità.

L'importanza della presenza di meticillino-resistenza è legata alla coesistenza di multiresistenze che limitano considerevolmente le opzioni terapeutiche.

In seguito all'incremento dell'incidenza di eventi epidemici causati in tutto il mondo da ceppi MRSA multiresistenti, i glicopeptidi sono sempre più spesso utilizzati per il trattamento delle infezioni stafilococciche nosocomiali.

Ciò ha contribuito allo sviluppo di isolati con ridotta sensibilità a tali farmaci (ceppi VISA ed h-VISA).

Da qui la necessità di ricercare, per ampliare l'armamentario terapeutico nei confronti dei patogeni Gram positivi, nuove molecole antibiotiche.

Il ceftaroline è un nuovo antimicrobico beta-lattamico di cui è stata approvata l'immissione in commercio nell'Agosto del 2012 per far fronte alle infezioni provocate da $S$. aureus.

Nello studio condotto su 80 stipiti di $S$. aureus, 50 dei quali MRSA e 30 MSSA, raccolti da ospedali del Nord Italia, ceftaroline ha dimostrato avere una buona attività inibente presentando MIC molto basse ed inferiori ai valori di MIC di altri antibiotici testati.

Il valore della MIC è un indice che dimostra l'alta affinità del ceftaroline per la PenicillinBinding-Protein $2 \mathrm{a}$.

L'antibiotico ha anche dimostrato attività battericida nei confronti di un ampio numero di stipiti di S. aureus, compresi gli isolati resistenti (MRSA, hVISA). Nel 96.25\% (77/80) dei casi i valori di MIC e di MBC coincidevano; in tre casi quest'ultima era superiore di una diluizione.

Pertanto anche i dati ottenuti in questo studio confermano che ceftaroline rappresenta una valida opzione terapeutica per il trattamento delle infezioni acquisite sia in ambito ospedaliero che comunitario sostenute da Staphylococcus aureus sia sensibile che resistente alla meticillina.

Tabella I. Criteri interpretivi di sensibilità per ceftaroline proposti da EUCAST

\begin{tabular}{cllcc}
\hline Microrganismo & MIC $(\mu \mathrm{g} / \mathrm{ml})$ & & Diametri degli aloni di inibizione (mm) \\
\hline & $\mathbf{S} \leq$ & $\mathbf{R}>$ & $\mathbf{S} \leq$ & $\mathbf{R}>$ \\
\hline S. aureus' & I & I & $\mathbf{2 0}$ & $\mathbf{2 0}$ \\
\hline
\end{tabular}

'Gli isolati MSSA possono essere riportati come sensibili a ceftaroline senza ulteriori test di sensibilità. Per gli isolati MRSA che presentano il diametro degli aloni di inibizione tra 19 e $21 \mathrm{~mm}$, si rende necessario determinare il corretto valore di MIC per confermarne la sensibilità. 
Tabella 2. Range dei valori di MIC degli stipiti di S. aureus MRSA di alcuni antibiotici attivi contro i batteri Gram-positivi. Valori di $\mathrm{MIC}_{50}$ e $M I C_{90}$ della cefalosporina. NR: Non Rilevato

\begin{tabular}{|c|c|c|c|c|}
\hline \multirow{2}{*}{ Organismo } & \multirow{2}{*}{ Antibiotico } & \multicolumn{3}{|l|}{ MIC ( $\mu \mathrm{g} / \mathrm{ml})$} \\
\hline & & Range & $\mathrm{MIC}_{50}$ & $\mathrm{MIC}_{90}$ \\
\hline \multirow{13}{*}{$\begin{array}{l}\text { S. aureus } \\
\text { (MRSA) }\end{array}$} & Ceftaroline & $0.125-2$ & 0.5 & 1 \\
\hline & Ampicillina & $>8$ & NR & NR \\
\hline & Clindamicina & $\leq 0.25->2$ & NR & NR \\
\hline & Daptomicina & $\leq 0.5-1$ & NR & NR \\
\hline & Eritromicina & $\leq 1->2$ & NR & NR \\
\hline & Fosfomicina & $\leq 32$ & NR & NR \\
\hline & Gentamicina & $\leq 1->4$ & NR & NR \\
\hline & Levofloxacina & $>2$ & NR & NR \\
\hline & Linezolid & $\leq 2-4$ & NR & NR \\
\hline & Oxacillina & $>2$ & NR & NR \\
\hline & Penicillina & $>2$ & NR & NR \\
\hline & Vancomicina & $\leq 0.5-2$ & NR & NR \\
\hline & Tetraciclina & $\leq 1->2$ & NR & NR \\
\hline
\end{tabular}

Tabella 3. Range dei valori di MIC negli stipiti di S. aureus MSSA e di alcuni antibiotici attivi contro i batteri Gram-positivi. Valori di $M I C_{50}$ e $M I C_{90}$ della cefalosporina. NR: Non Rilevato.

\begin{tabular}{|c|c|c|c|c|}
\hline \multirow[t]{2}{*}{ Organismo } & \multirow[t]{2}{*}{ Antibiotico } & \multicolumn{3}{|l|}{ MIC ( $\mu \mathrm{g} / \mathrm{ml})$} \\
\hline & & Range & $\mathrm{MIC}_{50}$ & MIC $_{90}$ \\
\hline \multirow{13}{*}{$\begin{array}{l}\text { S. aureus } \\
\text { (MSSA) }\end{array}$} & Ceftaroline & $0.06-0.5$ & 0.125 & 0.25 \\
\hline & Ampicillina* & $\leq 2->8$ & NR & NR \\
\hline & Clindamicina & $\leq 0.25-0.5$ & NR & NR \\
\hline & Daptomicina & $\leq 0.5-1$ & NR & NR \\
\hline & Eritromicina & $\leq 0.5->2$ & NR & NR \\
\hline & Fosfomicina & $\leq 32$ & NR & NR \\
\hline & Gentamicina & $\leq \mathrm{I}->4$ & NR & NR \\
\hline & Levofloxacina & $\leq 1-4$ & NR & NR \\
\hline & Linezolid & $\leq 2-4$ & NR & NR \\
\hline & Oxacillina & $\leq 0.25-1$ & NR & NR \\
\hline & Penicillina* & $\leq 0.12->8$ & NR & NR \\
\hline & Vancomicina & $1-2$ & NR & NR \\
\hline & Tetraciclina & $\leq 1->2$ & NR & NR \\
\hline
\end{tabular}

* 3/30 ceppi risultavano sensibili alla Penicillina.

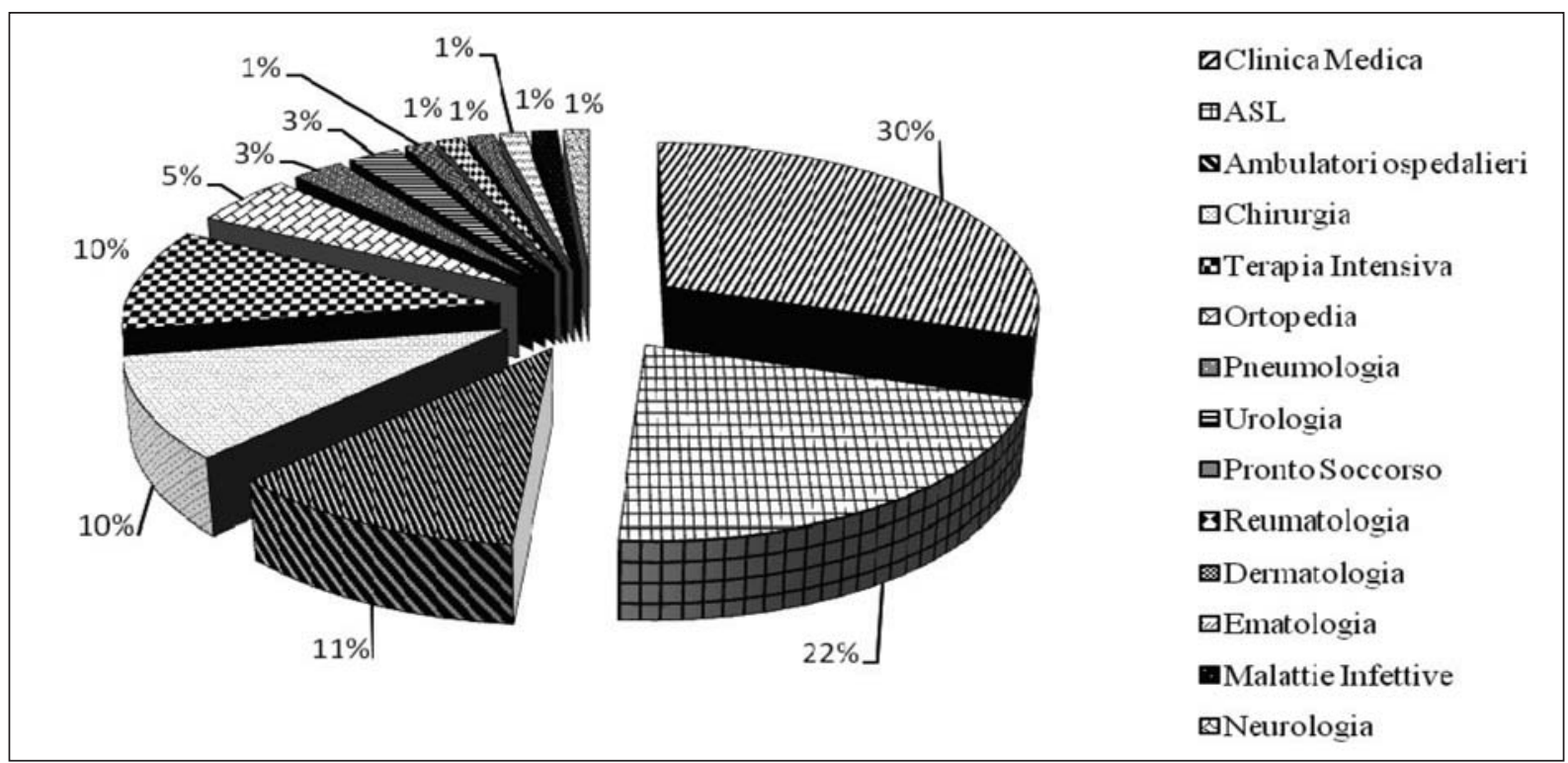

Figura I. Distribuzione per reparto degli 80 stipiti di S. aureus. 

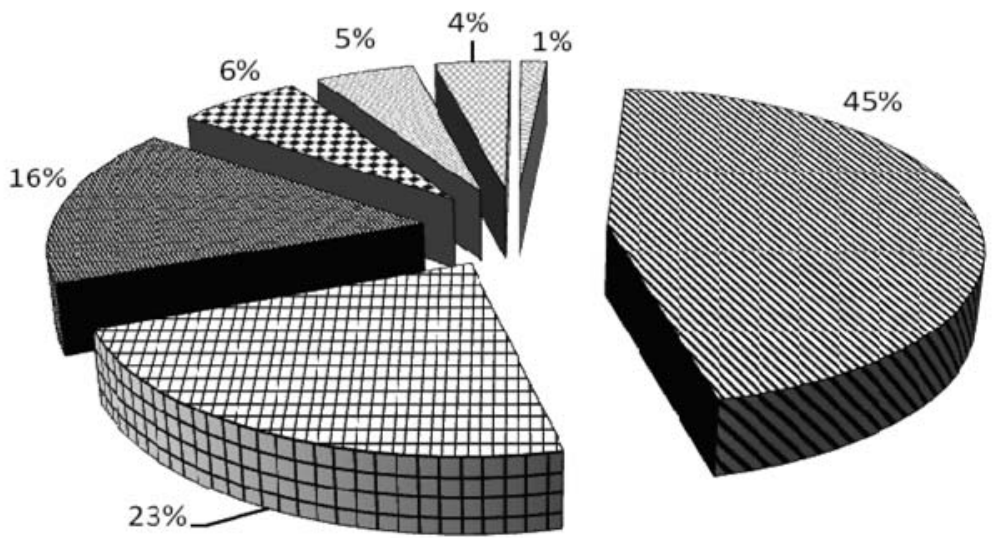

$\boldsymbol{\nabla}$ Tamponi ferita

$\square$ Emocoltura

圆 Broncoaspirato

I I iquidi

๑rine

๑Tamponi vari

口 Punta Cat.Ven.Centr.

Figura II. Distribuzione per materiale biologico degli 80 stipiti di S. aureus.

\section{BIBLIOGRAFIA}

1. Clinical and Laboratory Standards Institute. Methods for dilution antimicrobial susceptibility tests for bacteria that grow aerobically: approved standard, 9th ed. CLSI M07-A9. Clinical and Laboratory Standards Institute, Wayne, PA. 2012.

2. Ge Y, Biek D, Talbot GH, Sahm DF. In vitro profiling of ceftaroline against a collection of recent bacterial clinical isolates from across the United States. Antimicrob Agents Chemother 2008; 52(9): 3398-407.

3. Laudano JB. Ceftaroline fosamil: a new broadspectrum cephalosporin. J Antimicrob Chemother
2011; 66 Suppl 3: iii11-8.

4. Tenover FC, Arbeit RD, Goering RV, et al. Interpreting chromosomal DNA restriction patterns produced by pulsed-field gel electrophoresis: criteria for bacterial strain typing. J Clin Microbiol 1995; 33: 2233-9.

5. The European Committee on Antimicrobial Susceptibility Testing. Breakpoint tables for interpretation of MICs and zone diameters. Version 2.0, 2012. http://www.eucast.org.

6. The European Committee on Antimicrobial Susceptibility Testing. Breakpoint tables for interpretation of MICs and zone diameters. Version 3.1, 2013. http://www.eucast.org. 